\title{
WHITNEY NUMBER INEQUALITIES FOR GEOMETRIC LATTICES
}

\author{
THOMAS A. DOWLING ${ }^{1}$ AND RICHARD M. WILSON 2
}

ABSTRACT. Let $L$ be a finite geometric lattice of rank $r$, and for $i=0,1, \cdots, r$, let $W_{i}$ denote the number of elements of $L$ with rank $i$. For $1 \leq k \leq r-2$, we have $W_{1}+W_{2}+\cdots+W_{k} \leq W_{r-k}+\cdots+W_{r-2}+W_{r-1}$ with equality if and only if the lattice $L$ is modular. We give two further results concerning matchings of lattice elements of rank $\leq k$ into those of rank $\geq r-k$, and observe that a middle term can be interpolated in the above inequality.

1. Introduction. For a finite geometric lattice $L$ of rank $r$, we denote the number of lattice elements of rank $i$ by $W_{i}=W_{i}(L) . W_{0}, W_{1}, \cdots, W_{r}$ are the Whitney numbers of the second kind. Of course, $W_{0}=W_{r}=1$.

There are several interesting conjectures concerning the Whitney numbers of geometric lattices. Foremost among these is the unimodality conjecture of G.-C. Rota which asserts that $W_{j} \geq \min \left\{W_{i}, W_{k}\right\}$ whenever $i \leq j \leq k$. This is known to be true for partition lattices [9], [11]. Another conjecture asserts that $W_{k} \leq W_{r-k}$ whenever $k \leq r / 2$ in a geometric lattice of rank $r$. This would imply that $W_{0} \leq W_{1} \leq W_{2} \leq \cdots \leq W_{[r / 2]}$ (by applying the above conjecture to truncations of the lattice). All of these conjectures are valid for geometric lattices with at most eight points [2] and "perfect matroid designs", [13].

In [1], Kelly and Basterfield proved that $W_{1} \leq W_{r-1}$ for geometric lattices of rank $r$. Another proof is given by C. Greene [7] who added the result that $W_{1}=W_{r-1}$ holds if and only if the lattice is modular, and also gave several results concerning the matching of the points into the copoints. A third proof, by entirely different techniques, is given by Greene in [8].

In this note we prove that $W_{1}+w_{2}+\cdots+w_{k} \leq W_{r-k}+\cdots+W_{r-2}+W_{r-1}$

Presented to the Society, January 25, 1973 under the title $A$ Whitney number inequality for geometric lattices; received by the editors June 26, 1973 and, in revised form, December 13, 1973.

AMS (MOS) subject classifications (1970). Primary 05B35; Secondary 05A20,

1 This research supported in part by NSF Grant GP 38951 (O.S.U.R.F. Project No. 3684-A1).

2 This research supported in part by NSF Grant GP 28943 (O.S.U.R.F. Project No. 3228-A1). 
for geometric lattices of rank $r$ with equality holding for some $k, 1 \leq k \leq$ $r-2$, if and only if the lattice is modular. This provides some justification for the empirical observation that geometric lattices are "top heavy" and is not unexpected in view of Crapo and Rota's assertion [4] that "Roughly speaking... everything which 'happens' in a geometric lattice also 'happens' somewhere at the top of the lattice."

Our proof appeals to elementary linear algebra and Möbius inversion. The inequality is proved by showing that a certain set of $w_{r-k}+\cdots+W_{r}$ vectors spans a $\left(W_{0}+\cdots+W_{k}\right)$-dimensional subspace of a vector space $V(L)$ introduced in $\$ 3$. These techniques also allow easy derivations of the generalizations of Greene's results concerning matchings of points to copoints (Theorems 2 and 3 of $\$ 5$ ). In Theorem 4 of $\$ 5$, we give a further result to indicate possible variations of the methods. A corollary of this result is used as a lemma in a further paper [6] where the authors give lower bounds for $W_{k}$ in terms of $W_{1}$ and the rank $r$ of a geometric lattice.

2. Preliminaries. Definitions and results required in the sequel are summarized in this section. A detailed treatment of geometric lattices may be found in [3] or [4].

A geometric lattice is a lattice $L$ of finite height in which $x>y(x$ covers $y$ ) if and only if $x=y \vee p$ for some point $p \notin y y$. Here a point is a lattice element covering the minimum element 0 of $L$. The maximum element of a lattice $L$ is denoted by 1 , and a copoint is a lattice element covered by 1 .

The rank function $\rho$ of a geometric lattice $L$ is uniquely determined by

$$
\rho(0)=0 \text { and } \rho(x)=\rho(y)+1 \text { whenever } x>y,
$$

and satisfies the semimodular law

$$
\rho(x)+\rho(y) \geq \rho(x \vee y)+\rho(x \wedge y)
$$

A geometric lattice is modular when $\rho(x)+\rho(y)=\rho(x \vee y)+\rho(x \wedge y)$ for all $x, y \in L$. The rank of $L$ is the integer $r=\rho(1)$.

For $a \leq b$ in a geometric lattice, the interval $[a, b]=\{x \in L: a \leq x \leq b\}$ is also a geometric lattice. When $a \leq x \leq b$ in a geometric lattice, there always exist modular complements of $x$ in the interval $[a, b]$, i.e., lattice elements $y$ such that $x \wedge y=a, x \vee y=b$, and $\rho(x)+\rho(y)=\rho(a)+\rho(b)$.

The Möbius function [12] of a finite lattice $L$ is the integer-valued function $\mu=\mu_{L}$ on $L \times L$ with the properties $\mu(x, y)=0$ unless $x \leq y$, 
$\mu(x, x)=1$, and $\Sigma_{x \leq z \leq y} \mu(x, z)=\Sigma_{x \leq z \leq y} \mu(z, y)=0$ whenever $x<y$. From these properties follows the principle of Möbius inversion: Given functions $f, g$ from $L$ into an additive abelian group, $f(x)=\Sigma_{y \geq x} g(y)$. for all $x$ implies $g(x)=\Sigma_{y \geq x} \mu(x, y) f(y)$, and $f(x)=\Sigma_{y \leq x} g(y)$ implies $g(x)=$ $\Sigma_{y \leq x} \mu(y, x) f(y)$.

We shall require the fact [12, Theorem 3] that for a geometric lattice, $\mu(x, y) \neq 0$ for $x \leq y$.

In [5, Lemma 2.2], Dilworth showed that in a modular geometric lattice, $W_{k}=W_{r-k}$ for all $k$.

3. A vector space. Given a finite lattice $L$, we introduce the free vector space $V(L)$ (over the rationals $Q$ ) generated by the lattice elements. Formally, we may take $V(L)$ to be the set of all mappings from $L$ into $Q$, with the usual addition and scalar multiplication. For each lattice element $x$, let $I_{x} \in V(L)$ denote the characteristic function of the singleton subset $\{x\}$, i.e.,

$$
I_{x}(y)= \begin{cases}1 & \text { if } y=x \\ 0 & \text { otherwise. }\end{cases}
$$

Clearly, the vectors $\left\{I_{x}: x \in L\right\}$ form a basis for $V(L)$.

For each $x \in L$, let $J_{x}$ and $K_{x}$ denote, respectively, the characteristic functions of the subsets $\{y \in L: y \bigvee x=1\}$ and $\{y \in L: y \leq x\}$. That is,

$$
J_{x}=\sum_{y: y \bigvee x=1} I_{y} \text { and } K_{x}=\sum_{y: y \leq x} I_{y} .
$$

The linear relations between the three sets of vectors $\left\{I_{x}\right\},\left\{J_{x}\right\},\left\{K_{x}\right\}$ are given in the following lemma in terms of the Möbius function $\mu$ of the lattice $L$.

Lemma 1. Let $L$ be a finite lattice. Then for each $x \in L$, the following equations hold in $V(L)$ :

(i) $I_{x}=\Sigma_{y: y \leq x} \mu(y, x) K_{y}$.

(ii) $J_{x}=\Sigma_{y: y \geq x} \mu(y, 1) K_{y}$.

(iii) $\mu(x, 1) K_{x}=\Sigma_{y: y \geq x} \mu(x, y) J_{y}$.

(iv) If $\mu(a, 1) \neq 0$ for all $a \in L$, then

$$
I_{x}=\sum_{y} \lambda(x, y) J_{y} \quad \text { where } \lambda(x, y)=\sum_{a: a \leq x \wedge y} \frac{\mu(a, x) \mu(a, y)}{\mu(a, 1)} .
$$

Proofs. For (i), apply Möbius inversion to the definition of $K_{x}$. For (ii), observe that 


$$
\sum_{y: y \geq x} \mu(y, 1) K_{y}=\sum_{y: y \geq x} \mu(y, 1) \sum_{z: z \leq y} I_{z}=\sum_{z}\left(\sum_{y: y \geq x \vee z} \mu(y, 1)\right) I_{z}=J_{x}
$$

since

$$
\sum_{y: 1 \geq y \geq x \vee z} \mu(y, 1)= \begin{cases}1 & \text { if } x \vee z=1 \\ 0 & \text { otherwise }\end{cases}
$$

Applying Möbius inversion to (ii), we arrive at (iii).

Assuming $\mu(a, 1)$ is never zero, (i) and (iii) give

$$
I_{x}=\sum_{a: a \leq x} \mu(a, x) K_{a}=\sum_{a: a \leq x} \frac{\mu(a, x)}{\mu(a, 1)} \sum_{y: y \geq a} \mu(a, y) J_{y}=\sum_{y} \lambda(x, y) J_{y} .
$$

Remark. With $x=0$ in Lemma 1 (iii) and $0 \neq a \in L$,

$$
\mu(0,1) K_{0}(a)=\sum_{y} \mu(0, y) J_{y}(a), \quad \text { or } 0=\sum_{y: y \vee a=1} \mu(0, y) .
$$

This is one form of Weisner's theorem [12, p. 351].

4. The main theorem.

Theorem 1. For any finite geometric lattice $L$, the Whitney numbers satisfy

$$
W_{1}+W_{2}+\cdots+W_{k} \leq W_{r-k}+\cdots+W_{r-2}+W_{r-1} \text {, }
$$

where $r$ is the rank of $L$ and $1 \leq k \leq r-1$. If equality holds for some $k$, $1 \leq k \leq r-2$, then the lattice $L$ is modular.

Proof. Let $U_{k}$ be the subspace of $V(L)$ spanned by the vectors $\left\{I_{x}: \rho(x) \leq k\right\}$, and let $\pi: V(L) \rightarrow U_{k}$ be the projection associating to each mapping $L \rightarrow Q$ of $V(L)$ its restriction to the subset $\{x \in L: \rho(x) \leq k\}$. That is, $\pi$ is the linear mapping $V(L) \rightarrow U_{k}$ defined by

$$
\pi\left(I_{x}\right)= \begin{cases}I_{x} & \text { if } \rho(x) \leq k \\ 0 & \text { otherwise }\end{cases}
$$

Note that if $\rho(y)<r-k$, then

$$
\pi\left(J_{y}\right)=\sum_{x: x \vee y=1} \pi\left(I_{x}\right)=0
$$

since $x \vee y=1$ implies $\rho(x) \geq \rho(1)+\rho(x \wedge y)-\rho(y)>k$ by the semimodular law (1). 
For each $x \in L$ with $\rho(x) \leq k$, we have from Lemma 1(iv),

$$
I_{x}=\pi\left(I_{x}\right)=\sum_{y} \lambda(x, y) \pi\left(J_{y}\right)=\sum_{y: \rho(y) \geq r-k} \lambda(x, y) \pi\left(J_{y}\right) .
$$

It follows that the $W_{r-k}+\cdots+W_{r-1}+W_{r}$ vectors $\left\{\pi\left(J_{y}\right): \rho(y) \geq r-k\right\}$ span the subspace $U_{k}$ which has dimension $W_{0}+W_{1}+\cdots+W_{k}$. Since $W_{0}=$ $W_{r}=1$, the stated inequality (2) is established.

We now assume that equality holds in (2) for some $k, 1 \leq k \leq r-2$. Then, evidently, the vectors $\left\{\pi\left(J_{y}\right): \rho(y) \geq r-k\right\}$ form a basis for the subspace $U_{k}$. For $x \in L$ with $\rho(x)=k+1$, we have from Lemma 1(iv),

$$
0=\pi\left(I_{x}\right)=\sum_{y: \rho(y) \geq r-k} \lambda(x, y) \pi\left(J_{y}\right)
$$

and hence $\lambda(x, y)=0$ whenever $\rho(y) \geq r-k$.

Now $x \wedge y=0$ would imply that $\lambda(x, y)=\mu(0, x) \mu(0, y) / \mu(0,1) \neq 0$. Thus our assumption of equality implies in particular that $x \wedge y>0$ whenever $\rho(x)=k+1$ and $\rho(y)=r-k$. The proof of Theorem 1 is thus completed by the following lemma.

Lemma 2. Let $L$ be a geometric lattice of rank $r$ and $1 \leq k \leq r-2$. If $x \wedge y>0$ whenever $\rho(x)=k+1$ and $\rho(y)=r-k$, then the lattice $L$ is modular.

Proof. Consider first the case $k=1$. If $L$ is not modular, there exist elements $x, y \in L$ with $\rho(x)+\rho(y)>\rho(x \wedge y)+\rho(x \vee y)$. The image of a maximal chain $C$ in $[x \wedge y, y]$ under the order homomorphism $z \mapsto x \vee z$ is then a maximal chain in $[x, x \vee y]$ with repeated elements, so there must exist three consecutive members $z_{1} \lessdot z_{2} \lessdot z_{3}$ of $C$ with $x \vee z_{1} \lessdot x \vee z_{2}=$ $x \vee z_{3}$. We may therefore assume without loss of generality (replace $x$ by $x \vee z_{1}, y$ by $\left.z_{3}\right)$ that $\rho(x \vee y)-\rho(x)=1, \rho(y)-\rho(x \wedge y)=2$. Let $c$ be a modular complement of $x \vee y$ in the interval $[x, 1]$ and let $l$ be a modular complement of $x \wedge y$ in the interval $[0, y]$. Then $\rho(c)=r-1, \rho(l)=2$ and

$$
l \wedge c=l \wedge y \wedge c=l \wedge y \wedge(x \vee y) \wedge c=l \wedge y \wedge x=0
$$

contrary to hypothesis.

Consider now the general case. The hypothesis remains unchanged if we replace $k$ by $r-k-1$, so we shall assume $k \leq r / 2$.

Suppose for contradiction, that $L$ is not modular. Then there exists a copoint $c$ and a line $l$ such that $l \wedge c=0$. Choose $z \geq l$ with $\rho(z)=k+1$ and put $a=z \wedge c$. We have $\rho(a) \leq k$. There are two cases. 
Case 1. $\rho(a) \leq k-1$. Let $y$ be a modular complement of $a$ in the interval $[0, c]$. Then $\rho(y) \geq r-k$, and $z \wedge y=z \wedge c \wedge y=a \wedge y=0$, a contradiction.

Case 2. $\rho(a)=k$. Choose $b \geq z$ with $\rho(b)=2 k$ and let $x$ be a modular complement of $z$ in the interval $[l, b]$. Let $y$ be a modular complement of $b$ in $[a, 1]$. Then $\rho(x)=k+1, \rho(y)=r-k$, and

$$
x \wedge y=x \wedge b \wedge y=x \wedge a=x \wedge z \wedge c=l \wedge c=0
$$

contrary to hypothesis.

5. Further results.

Theorem 2. Let $L$ be a finite lattice such that $\mu(a, 1) \neq 0$ for all $a \in L$. Then there exists a permutation $f: L \rightarrow L$ such that $x \vee f(x)=1$ for all $x \in L$.

Proof. By Lemma 1 (iv), $\left\{J_{x}: x \in L\right\}$ is a basis for $V(L)$. Hence the matrix whose rows and columns are indexed by $L$, the entry in row $x$ and column $y$ being

$$
J_{x}(y)= \begin{cases}1 & \text { if } x \vee y=1 \\ 0 & \text { otherwise }\end{cases}
$$

is nonsingular. Some term in the determinant expansion does not vanish, i.e. for some permutation $f$ of $L, J_{x}(f(x))=1$ for all $x \in L$.

(Note that, necessarily, $f(0)=1$ and $f(1)=0$.)

Remark. The inequality of Theorem 1 is an immediate consequence of Theorem 2 since for a geometric lattice $L, f$ maps elements of rank $\leq k$ injectively into elements of rank $\geq r-k$.

By applying Theorem 2 to the dual lattice $L^{*}$, we have

Theorem $2 *$. Let $L$ be a finite lattice such that $\mu(0, a) \neq 0$ for all $a \in L$. Then there exists a permutation $f^{*}: L \rightarrow L$ such that $f^{*}(0)=1$, $f^{*}(1)=0$, and $x \wedge f^{*}(x)=0$ for all $x \in L$.

This answers affirmatively a conjecture of D. G. Kelly [10] that the assertion of Theorem $2 *$ holds for geometric lattices.

An example of a nongeometric lattice for which Theorems 2 and $2 *$ apply is the lattice of faces of a convex polytope, where $\mu(E, F)$ is $(-1)^{\operatorname{dim}(F)-\operatorname{dim}(E)}$ for faces $E \leq F$. 
Theorem 3. Let $L$ be a finite geometric lattice of rank $r$ and $0 \leq k \leq r$. Then there exists an injection $g:\{x \in L: \rho(x) \leq k\} \rightarrow\{y \in L: \rho(y) \geq r-k\}$ such that $x \leq g(x)$ for all $x$ in the domain of $g$.

Proof. With the notation as in the proof of Theorem 1, we have seen that $\left\{\pi\left(J_{y}\right): \rho(y) \geq r-k\right\}$ spans the subspace $U_{k}$. By Lemma 1 (ii),

$$
\pi\left(J_{y}\right)=\sum_{z: z \geq y} \mu(z, 1) \pi\left(K_{z}\right)
$$

so, evidently, $\left\{\pi\left(K_{z}\right): \rho(z) \geq r-k\right\}$ also spans $U_{k}$. Hence the matrix whose rows are indexed by $\{x \in L: \rho(x) \leq k\}$ and columns by $\{y \in L: \rho(y) \geq r-k\}$, the entry in row $x$ and column $y$ being

$$
K_{y}(x)= \begin{cases}1 & \text { if } x \leq y \\ 0 & \text { otherwise }\end{cases}
$$

has rank equal to the number of rows. The existence of the injection $g$ now follows, since some maximal square submatrix is nonsingular.

Remark. From Lemma 1 and with $T=\{y \in L: \rho(y) \geq r-k\}$,

$$
\mu(0,1) I_{0}=\sum_{y \in T} \mu(0, y) \pi\left(J_{y}\right)=\sum_{z}\left(\sum_{y \leq z, y \in T} \mu(0, y)\right) \mu(z, 1)_{\pi}\left(K_{z}\right) .
$$

The coefficient of $\mu(0,1) \pi\left(K_{1}\right)$ in this sum is $\Sigma_{y \in T} \mu(0, y)=\mu_{L^{\prime}}(0,1)$, where $L^{\prime}$ is the $k$ th truncation of $L$, i.e. the geometric lattice obtained by identifying all elements of $L$ with rank $\geq r-k$. In particular, the coefficient of $\pi\left(K_{1}\right)$ is nonzero. This remark can be used to see that in Theorem 3, the injection $g$ can be chosen so that $g(0)=1$ (whenever $k<r$ ).

For a geometric lattice $L$, let $\operatorname{Top}_{k}(L)$ and $\operatorname{Bot}_{k}(L)$ denote, respectively, the sum of the top and bottom $k+1$ Whitney numbers of $L$, i.e.

$$
\begin{aligned}
& \operatorname{Bot}_{k}(L)=W_{0}+W_{1}+\cdots+W_{k}, \\
& \operatorname{Top}_{k}(L)=W_{r}+W_{r-1}+\cdots+W_{r-k}, \quad \text { where } r=\operatorname{rank}(L) .
\end{aligned}
$$

Theorem 4. Let $L$ be a finite geometric lattice of rank $r$ and fix $a \in L$ with $\rho(a) \leq r-k$. Then

$$
\operatorname{Top}_{k}(L) \geq \operatorname{Top}_{k}(L)-\operatorname{Top}_{k}([a, 1])+\operatorname{Bot}_{k}([a, 1]) \geq \operatorname{Bot}_{k}(L)
$$

Proof. Applying Theorem 1 to the geometric lattice $[a, 1]$, we have 
$\operatorname{Top}_{k}([a, 1]) \geq \operatorname{Bot}_{k}([a, 1])$ and the first inequality follows.

For $z \in L$, define $A_{z}=\Sigma_{x: x \vee a=z} I_{x} \in V(L)$. Then whenever $y \geq a$ in $L$,

$$
K_{y}=\sum_{z: a \leq z \leq y} A_{z}
$$

To prove the second inequality, it will suffice to show that the set of vectors

$$
S=\left\{\pi\left(K_{y}\right): \rho(y) \geq r-k, y \geq a\right\} \cup\left\{\pi\left(A_{z}\right): \rho(z) \leq \rho(a)+k, z \geq a\right\}
$$

spans the subspace $U_{k}$ of $V(L)$. From the proof of Theorem 3, $\left\{\pi\left(K_{y}\right): \rho(y) \geq r-k\right\}$ spans $U_{k}$, so it remains only to show that $\pi\left(K_{y}\right)$ is in the span of $S$ for $y \geq a$. But this follows from equation (3) above and the observation that the semimodular law (1) implies $\pi\left(A_{z}\right)=0$ whenever $\rho(z)>$ $\rho(a)+k$.

With $k=1$ and $a$ taken to be a point, we have the

Corollary. In a finite geometric lattice of rank $\geq 2$, the number of lines (rank 2 lattice elements) on a given point plus the number of copoints not on that point cannot be less than the total number of points.

\section{REFERENCES}

1. J. G. Basterfield and L. M. Kelly, A characterization of sets of $n$ points which determine $n$ hyperplanes, Proc. Cambridge Philos. Soc. 64 (1968), 585-588. MR 38 \#2040.

2. J. E. Blackburn, H. H. Crapo and D. A. Higgs, A catalogue of combinatorial geometries, University of Waterloo, Waterloo, Ontario, 1969.

3. G. Birkhoff, Lattice theory, 3rd ed., Amer. Math. Soc. Colloq. Publ., vol. 25, Amer. Math. Soc., Providence, R. I., 1967. MR 37 \#2638.

4. H. H. Crapo and G.-C. Rota, On the foundations of combinatorial theory: Combinatorial geometries, M. I. T. Press, Cambridge, Mass., 1970. (preliminary edition). MR $45 \# 74$.

5. R. P. Dilworth, Proof of a conjecture on finite modular lattices, Ann. of Math. (2) 60 (1954), 359-364. MR 16, 106.

6. T. A. Dowling and R. M. Wilson, The slimmest geometric lattices, Trans. Amer. Math. Soc. 196 (1974), 203-215.

7. C. Greene, A rank inequality for finite geometric lattices, J. Combinatorial Theory 9 (1970), 357-364. MR $42 \# 1727$.

8. - Inequalities for geometric lattices, Proc. Conf. on Möbius Algebras (H. Crapo and G. Roulet, editors), University of Waterloo, Waterloo, Ont., 1971.

9. L. H. Harper, Stirling behaviour is asymptotically normal, Ann. Math. Statist. 38 (1967), 410-414. MR 35 \#2312.

10. D. G. Kelly, Disjoining permutations in finite boolean algebras, Utilitas Mathematica 3 (1973), 65-74. 
11. E. Lieb, Concavity properties and a generating function for Stirling numbers, J. Combinatorial Theory 5 (1968), 203-206. MR 37 \#6195.

12. G.-C. Rota, On the foundations of combinatorial theory. I: Theory of Möbius functions, Z. Wahrscheinlichkeitstheorie und Verw. Gebiete 2 (1964), 340-368. MR 30 \#4688.

13. P. Young, U. S. R. Murty and J. Edmonds, Equicardinal matroids and matroid designs, Proc. Second Chapel Hill Conference on Combinatorial Mathematics and its Applications, University of North Carolina, Chapel Hill, N. C., 1970.

DEPARTMENT OF MATHEMATICS, OHIO STATE UNIVERSITY, COLUMBUS, OHIO 43210 